\title{
Introduction of the in-orbit test and its performance for the first meteorological imager of the Communication, Ocean, and Meteorological Satellite
}

\author{
D. H. Kim $^{1}$ and M. H. Ahn ${ }^{2}$ \\ ${ }^{1}$ National Meteorological Satellite Center/KMA, 64-18 Guam-gil, Gwanghyewon-myeon, Jincheon-gun, \\ Chungcheongbuk-do, 365-830, Republic of Korea \\ ${ }^{2}$ Department of Atmospheric Science and Engineering, Ewha Womans University, 52 Ewhayeodae-gil, Seodaemun-gu, \\ Seoul, 120-750, Republic of Korea
}

Correspondence to: M. H. Ahn (terryahn65@ewha.ac.kr)

Received: 13 August 2013 - Published in Atmos. Meas. Tech. Discuss.: 17 December 2013

Revised: 20 June 2014 - Accepted: 26 June 2014 - Published: 12 August 2014

\begin{abstract}
The first geostationary Earth observation satellite of Korea - the Communication, Ocean, and Meteorological Satellite (COMS) - was successfully launched on 27 June 2010. After arrival at its operational orbit, the satellite underwent an in-orbit test (IOT) that lasted for about 8 months. During the IOT period, the main payload for the weather application, the meteorological imager, went through successful tests for demonstrating its function and performance, and the test results are introduced here.

The radiometric performance of the meteorological imager (MI) is tested by means of signal-to-noise ratio (SNR) for the visible channel, noise-equivalent differential temperature (NEdT) for the infrared channels, and pixel-to-pixel nonuniformity for both the visible and infrared channels. In the case of the visible channel, the SNR of all eight detectors is obtained using the ground-measured parameters with the background signals obtained in orbit. The overall performance shows a value larger than 26 at $5 \%$ albedo, exceeding the user requirement of 10 by a significant margin. Also, the relative variability of detector responsivity among the eight visible channels meets the user requirement, showing values within $10 \%$ of the user requirement. For the infrared channels, the NEdT of each detector is well within the user requirement and is comparable with or better than the legacy instruments, except for the water vapor channel, which is slightly noisier than the legacy instruments. The variability of detector responsivity of infrared channels is also below the user requirement, within $40 \%$ of the requirement, except for
\end{abstract}

the shortwave infrared channel. The improved performance result is partly due to the stable and low detector temperature obtained due to spacecraft design, i.e., by installing a single solar panel on the opposite side of the MI.

\section{Introduction}

Geostationary meteorological satellites have played important roles as storm trackers, a global source of important geophysical information such as sea surface temperature, and providers of long-term records for climatic applications (Purdom and Menzel, 1996; Schmetz et al., 2002). The current constellation of six geostationary satellites (GOES-W and GOES-E, MTSAT, FY-2, Meteosat, and Meteosat Indian Ocean) meets the basic requirements - a geostationary image at least twice an hour - although better temporal resolution is required for rapidly changing phenomena such as tropical cyclones. Furthermore, backup satellites for contingency situations are quite important to ensure the continuity of operational weather satellites (CGMS, 2007). In such situations, a new geostationary satellite capable of operational meteorological observation could play an important role.

The first multi-purpose geostationary observation satellite of Korea, COMS (Communication, Ocean, and Meteorological Satellite), is such an addition. COMS, successfully launched on 27 June 2010, is designed to perform three major missions, including operational weather observation along 


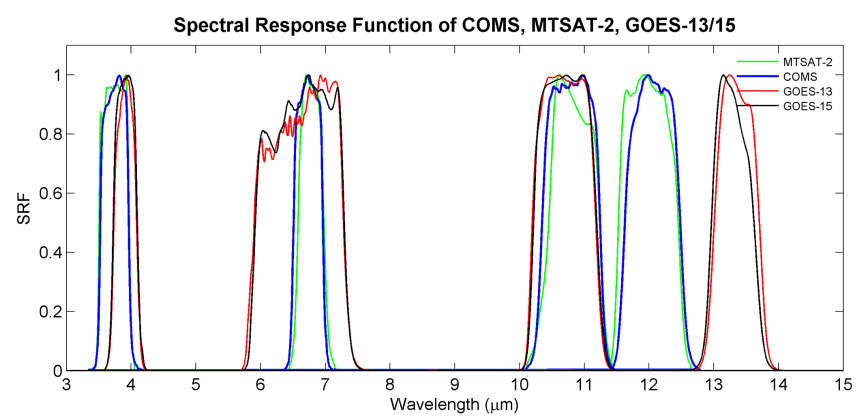

Figure 1. Comparison of the spectral response function (SRF) of COMS/MI with other legacy instruments onboard GOES-13/-15 and MTSAT-2 satellites.

with oceanography observation and space proofing of a Kaband transponder. The main payload for weather observation is the meteorological imager (MI), an imaging radiometer with the five observation channels, one in the visible band (VIS), one in shortwave infrared band (SWIR), one in the water vapor absorption band (WV), and two split window bands (IR1 and IR2). Figure 1 and Table 1 summarize the spectral response functions (SRFs) and the channel specifications, respectively. A more detailed description of COMS/MI is found in Appendix A1.

The COMS program has a few differences that stand out from legacy programs. For example, the planned mission lifetime of COMS is longer than that of usual geostationary meteorological satellite missions: 7 years versus 5 years. Also, the spacecraft is designed to provide the least interference with MI performance, especially for the infrared channels, due to it having a single solar panel without the balancing structure used for the previous GOES series or MTSAT series (Menzel and Purdom, 1994; SS/Loral, 1996). Thus, instrument characterization during the ground tests and the in-orbit test (IOT) performed after the launch are quite important for the new system and will play an important role in ensuring the longer mission lifetime. Here, we introduce the IOT procedures as well as the important outputs for the main meteorological payload, in terms of its functional and radiometric performances. Section 2 briefly summarizes the IOT procedures and results, followed by the characteristics of the radiometric performance of COMS/MI in Sect. 3. Section 4 summarizes the performance characteristics obtained during the IOT and concludes the paper.

\section{In-orbit test (IOT)}

Here, we introduce the activities and major outcomes from the IOT, started soon after COMS' arrival at the service orbit, $128.2^{\circ} \mathrm{E}$. The major goals for the IOT were to make sure that COMS and its payloads have survived the launch, to demonstrate that the performance in space is in line with the predicted performance, and to collect information and data

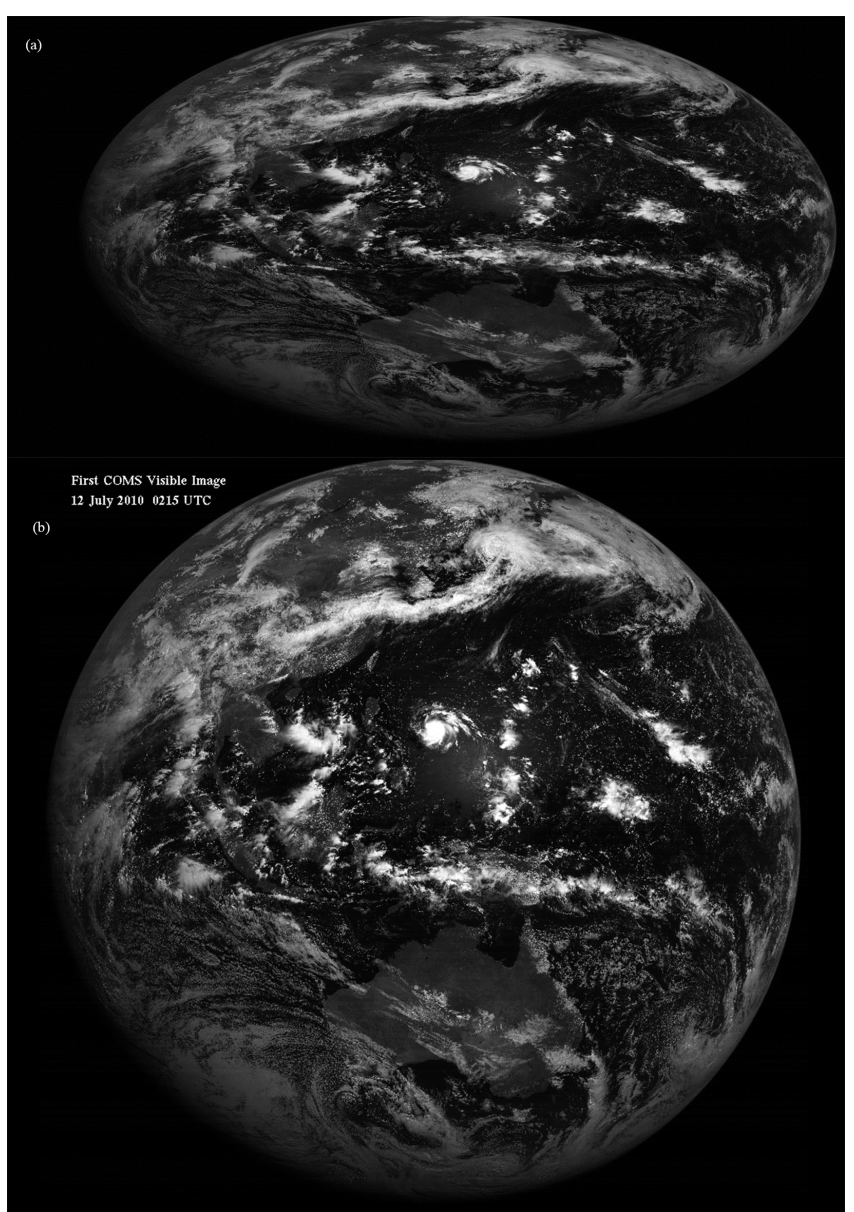

Figure 2. The first visible images of COMS/MI taken on 12 July 2010. (a) is the original 1.75 oversampled image and (b) is the rectified image.

for the actual operation of satellite, as well as fine tuning of equipment and algorithms. The IOT activities can be categorized into the activities for the system and payload checks, the functional and performance tests, and preparation for operation. While a detailed description for the IOT is provided in Appendix A2, several interesting activities such as the acquisition of the first image, a successful outgassing operation, and the beginning of the full test are introduced here.

The first COMS/MI image is taken from 02:15 UTC to 02:45 UTC on 12 July 2010, one day after the successful arrival at the service orbit. Figure 2 shows the original and rectified images of the first full-disk visible channel. The original image, which has not undergone any image navigation or radiometric calibration, is oversampled by a factor of 1.75 , while the rectified image is obtained by re-sampling of the original image. At the center of the image, the second tropical cyclone of the year, Conson, is clearly captured, with a bright circle of sun glint which happens to be in the southeast of the typhoon. Also, another important meteorological feature, the Asian monsoon, is clearly visible from the east-west (E-W) 
Table 1. Specifications of COMS/MI user requirement. Note that the dynamic range of SWIR goes up to $350 \mathrm{~K}$ to accommodate the daytime contribution of solar radiation.

\begin{tabular}{|c|c|c|c|c|c|c|}
\hline Channel & $\begin{array}{r}\text { Center wavelength } \\
(\mu \mathrm{m})\end{array}$ & BWHM & $\begin{array}{r}\text { Dynamic } \\
\text { range }\end{array}$ & $\begin{array}{l}\text { IFOV } \\
(\mu \mathrm{rad})\end{array}$ & $\begin{array}{r}\text { GSD } \\
(\mathrm{km})\end{array}$ & Noise \\
\hline VIS & 0.675 & $0.55-080$ & $0-115 \%$ & 28 & 1 & $\begin{array}{l}\mathrm{SNR}>170: 1 \text { at } 100 \% \text { albedo } \\
\mathrm{SNR}>10: 1 \text { at } 5 \% \text { albedo }\end{array}$ \\
\hline SWIR & 3.75 & $3.5-4.0$ & $4-350 \mathrm{~K}$ & 112 & 4 & $\begin{array}{l}\mathrm{NEdT}<0.10 \mathrm{~K} \mathrm{rms} \text { at } 300 \mathrm{~K} \\
\mathrm{NEdT}<5.7 \mathrm{~K} \mathrm{rms} \text { at } 220 \mathrm{~K}\end{array}$ \\
\hline WV & 6.75 & $6.5-7.0$ & $4-330 \mathrm{~K}$ & 112 & 4 & $\begin{array}{l}\mathrm{NEdT}<0.12 \mathrm{~K} \mathrm{rms} \text { at } 300 \mathrm{~K} \\
\mathrm{NEdT}<0.85 \mathrm{~K} \mathrm{rms} \text { at } 220 \mathrm{~K}\end{array}$ \\
\hline WIN1 & 10.8 & $10.3-11.3$ & $4-330 \mathrm{~K}$ & 112 & 4 & $\begin{array}{l}\mathrm{NEdT}<0.12 \mathrm{~K} \mathrm{rms} \text { at } 300 \mathrm{~K} \\
\mathrm{NEdT}<0.40 \mathrm{~K} \mathrm{rms} \text { at } 220 \mathrm{~K}\end{array}$ \\
\hline WIN2 & 12.0 & $11.5-12.5$ & $4-330 \mathrm{~K}$ & 112 & 4 & $\begin{array}{l}\mathrm{NEdT}<0.20 \mathrm{~K} \mathrm{rms} \text { at } 300 \mathrm{~K} \\
\mathrm{NEdT}<0.48 \mathrm{~K} \mathrm{rms} \text { at } 220 \mathrm{~K}\end{array}$ \\
\hline
\end{tabular}

VIS: visible; SWIR: shortwave infrared; WV: water vapor; WIN1: window 1; WIN2: window 2; BWMH: bandwidth at half maximum; GSD: ground sampling distance (in kilometers).

cloud bands which extend from southern China, through Korea, and over to Japan. Successful acquisition and the process of the first image proves the integrity of the whole signal flow, from the radiance measurement by COMS/MI, through onboard signal processing and transmission, to the reception and processing of signals at the ground station.

The first infrared images are taken much later than the first visible image, due to the outgassing operation, which essentially bakes out the instrument to minimize particle contamination on the surface of the sensitive optical surfaces. The coldest parts of MI such as the infrared detectors are susceptible to contamination. To minimize contamination of the sensitive parts of the instrument, special procedures are put in place. For example, during the launch and orbit-raising period, the coldest part is covered by the cooler cover. Also, as soon as possible, the outgassing mode is switched on to heat up the infrared detector suite and passive cooler to bake out any contaminants from the surface of these parts. In the case of the COMS IOT, this lasted about 6 weeks (same amount of time as the typically recommended 42 days) from launch date to 11 August 2010. COMS/MI can also be operated in such a mode, but no valid data are received from the IR channels.

As soon as the outgassing period ends, COMS/MI radiant cooler is deployed into operational mode (i.e., the passivecooler cover is opened and the cover is attached to the side of COMS/MI). With the opening of cooler cover, COMS/MI radiant cooler cools down the detector patch to a stabilized, regulated patch temperature. It takes about one day to finish the cooling and stabilization processes, and then the acquisition of the infrared images is started. The first infrared images were taken on 11 August, and the first images of four infrared channels are shown in Fig. 3. From the two window channels (Fig. 3c and b), several prominent cloud patterns associated with important weather processes such as tropical convective activities (center of image), weakened Asian monsoon (upper part of the image), and well-developed polar cyclones in the Southern Hemisphere are clearly captured. The water vapor image (Fig. 3d) reveals the upper air flow associated with those prominent cloud patterns. Finally, the SWIR image shows its characteristic features of strong temperature sensitivity (for example, upper-left part of the image is much darker (i.e., warmer) than other parts of image due to the effect of weak solar radiation). Overall, the first trial to acquire all five channel images was quite a success.

After the successful start of full operation of COMS/MI, the IOT is resumed for all five channels. The most important tasks with full operation are the fine tuning of the imagenavigation-and-registration (INR) algorithms and extensive verification of performances at system level, i.e., INR validation, band-to-band registration, and navigation performance. During this period, the simulated algorithm parameters for the INR processes are fine-tuned with the actual observation data and all of the algorithm parameters are finalized. The original plan for the length of the IOT was about 6 months; however, it took about 8 months, which is still considered quite successful compared to other similar programs.

\section{Radiometric performance}

The test results for the radiometric performance demonstrates that COMS/MI satisfies the end-user requirements by a significant margin for most of requirements. Here, we introduce the performance results of COMS/MI in terms of noise performance and detector-to-detector variation for both the visible and infrared channels. The noise performance is presented as the signal-to-noise ratio (SNR) for the visible channel and noise-equivalent differential temperature (NEdT) for the infrared channels. Pixel-to-pixel response nonuniformity (PRNU) provides the degree of differential responsivity for different detectors. The noise and PRNU are evaluated from the space look data, which are obtained by looking at the 
(a)

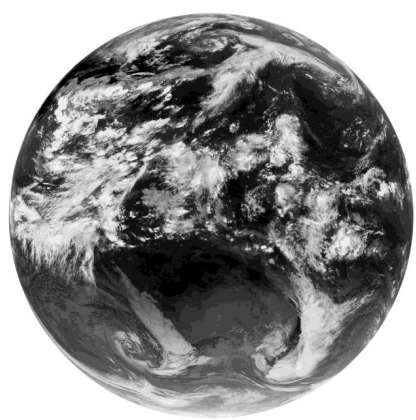

(c)

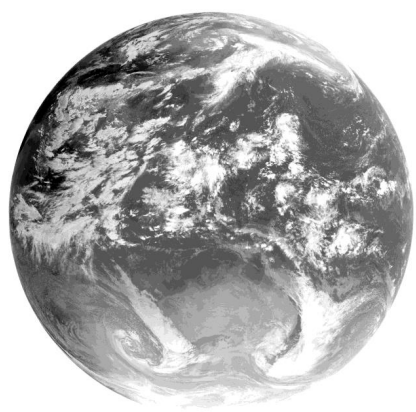

(b)

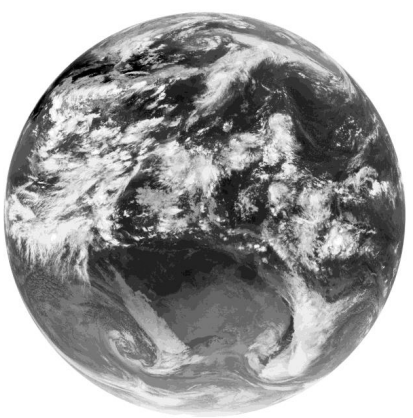

(d)

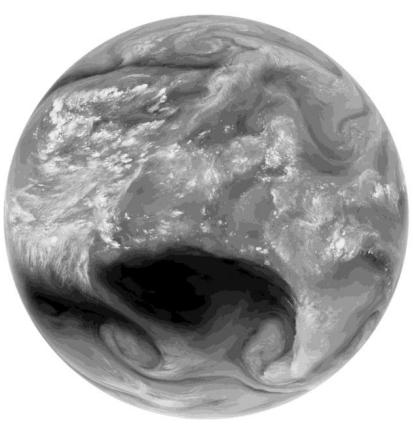

Figure 3. The first infrared images of COMS/MI taken $11 \mathrm{Au}-$ gust 2010. (a), (b), (c), and (d) are for the IR1, IR2, SWIR, and WV channels, respectively.

space scene away from the Earth scene with the calibration parameters obtained during the ground test of the instrument. The space look happens when either a full-disk observation is made or with a specific ground command.

To make sure that the selected Instantaneous field of views (IFOVs) for the performance tests are free of interference from the Earth, Moon, or Sun, the space look scene is carefully selected. This is also important for infrared channel calibration, because the space look data are used for background bias correction, which should be applied before derivation of the calibration coefficients (Weinreb et al., 1997). To have stable statistical outputs and to minimize any unexpected interference, the sensitivity of the background count values as a function of selected IFOV number and location is analyzed. Figure 4 shows an example of such a sensitivity test, which shows the mean and standard deviation of a detector count value of eight different visible detectors, with the different number of IFOVs selected to calculate the mean and standard deviation. From the test, we found that the background count value is quite stable from about 100 IFOVs away from the edge of image. Based on these test results, we choose $100 \times 100$ IFOVs selected from 100 IFOVs away from the upper and left or right edge of the image.

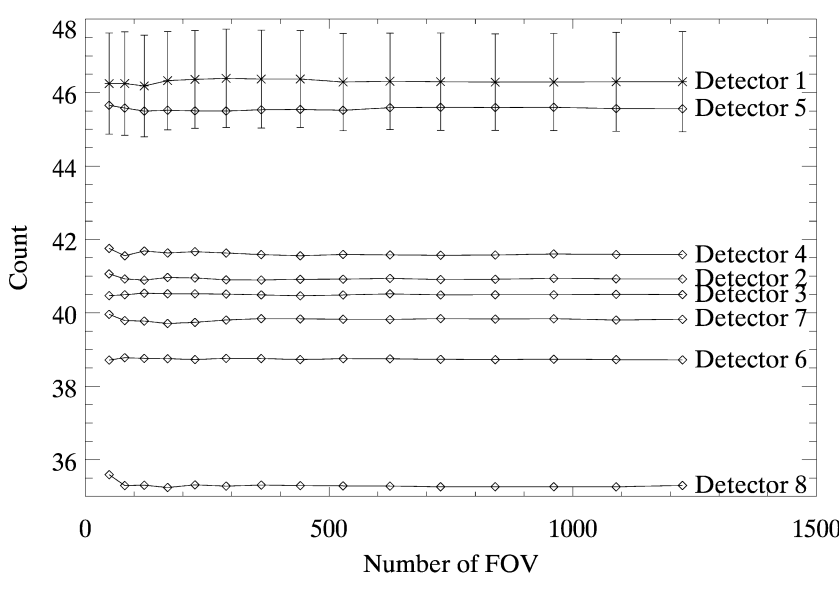

Figure 4. Variation of average and standard deviation of the space look signal in terms of the digital count value as a function of number of pixels used to estimate the average and standard deviation.

\subsection{Visible channel}

The SNR requirement for the visible channel of COMS/MI is referenced at both 5 and $100 \%$. In view of the quantitative use of the visible radiances taken over surfaces with low reflectivity, the requirement at $5 \%$ is quite important. This is specifically important for the quantitative derivation of aerosol optical depth, such as for Asian dust, using the singlechannel data (Kim et al., 2007). The SNR at $5 \%$ albedo is estimated using the Eq. (1) (Astrium EADS, 2011a):

$\mathrm{SNR}_{5} \%=\frac{R_{5} \%}{\sqrt{A_{\text {in_orbit }}+B_{\text {on_ground }} \times R_{5} \%}}$,

where $R_{5} \%$ is the radiance at $5 \%$ albedo; $A_{\text {in_orbit }}$ is the square of the noise radiance obtained from the space look; and $B_{\text {on_ground }}$ is the correction coefficient obtained from the ground tests conducted before the launch, estimated to be about 0.0021 (in units of radiance). The calculated $R_{5} \%$ varies mainly with the SRF of the specific channels, and the applied value for the COMS visible channels is estimated to be $23.92 \mathrm{~W} \mathrm{~m}^{-2} \mathrm{sr}^{-1} \mu \mathrm{m}^{-1}$. Thus the in-orbit SNR value is mainly determined by $A_{\text {in_orbit }}$, which is in turn obtained by the relationship of $A_{\text {in_orbit }}=\left(m \times \sigma_{\mathrm{c}}\right)^{2}$, where $m$ is the slope for conversion from count value to radiance of each visible detector and $\sigma_{\mathrm{c}}$ is the standard deviation of dark count values obtained from the space look counts. The slope is obtained during the ground test and differs for each detector. For example, the $m$ value for detector number 1 is about 0.594, while it is about 0.632 for detector number 5 (Astrium EADS, 2011a).

The space look count values obtained from the selected IFOVs are processed using Eq. (1), and the monthly mean SNR values for all eight detectors from August 2010 to March 2011 are summarized in Fig. 5, along with the diurnal variation of the time-averaged SNR values. At the beginning 
(a) Monthly mean SNR from August 2010 to March 2011

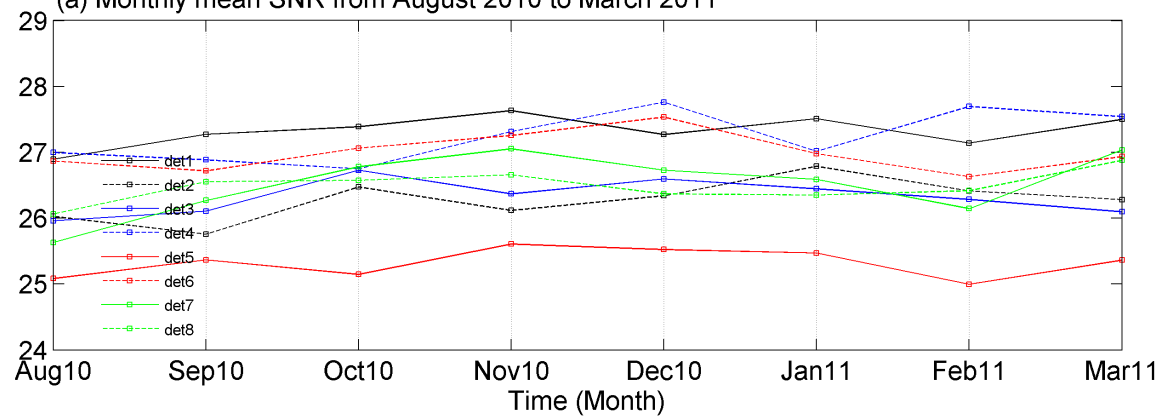

(b) Diurnal variation of SNR

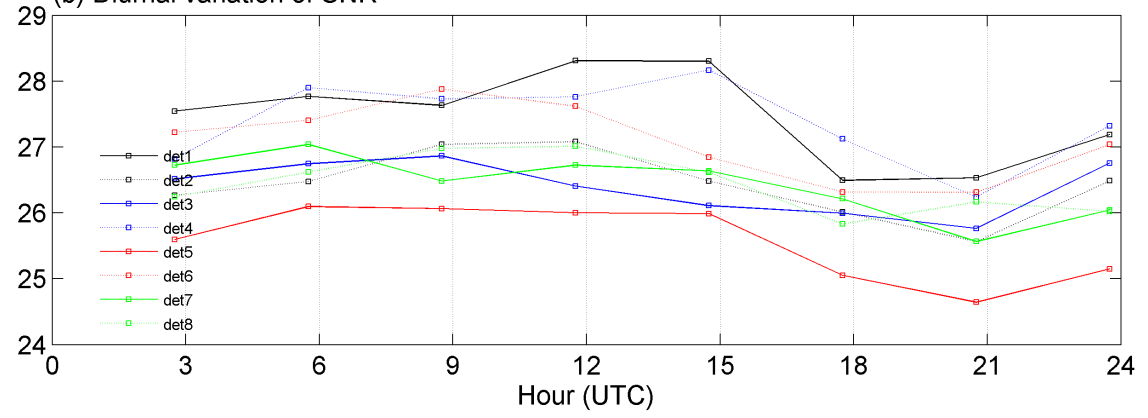

Figure 5. Time series of SNR of visible channels. (a) Monthly mean SNR of eight detectors from August 2010 to March 2011 . (b) Diurnal variation of SNR for full-disk images (every $3 \mathrm{~h}$ interval of measurement schedule for COMS/MI).

of the IOT, all eight SNR values are higher than the user requirement of 10. Among the eight detectors, detector number 1 has a slightly higher SNR value than that of detector number 5 , i.e., by about 2 . The difference is mainly due to the difference in the $m$ value used for the estimation of $A_{\text {in_orbit }}$. Although it looks to be a rather significant difference, the difference is compensated for by taking out the space look count during the calibration process, and it does not introduce significant consequences such as the PRNU value. In terms of the long-term trend, at least during the IOT period, all eight detectors show quite stable SNR performance, showing neither significant drift nor degradation, although there is small monthly variation, which is of almost the same magnitude as the uncertainty in the monthly mean SNR value, about 0.8. For the derivation of the diurnal variation shown in Fig. 5b, the full-disk images taken every $3 \mathrm{~h}$ interval are used. Contrary to the monthly mean values, there is a rather outstanding diurnal variation, showing the large SNR value during the satellite nighttime and relatively small value during the early morning hours. The diurnal variation is thought to be mainly due to the relative geometry of the Sun and instrument viewing angle.

Table 2 summarizes the overall performance of the SNR for the eight visible detectors at $5 \%$ albedo obtained by taking the average of all the available data obtained during the IOT period. The mean background count values obtained during the IOT are almost the same (maximum difference
Table 2. The mean and standard deviation $(\sigma)$ of space look count and the derived signal-to-noise ratio (SNR) at $5 \%$ albedo (reference band radiances for albedo of $5 \%$ is $23.92 \mathrm{~W} \mathrm{~m}^{-2} \mathrm{sr}^{-1} \mu \mathrm{m}^{-1}$ ). The user requirement for SNR of 10 is given at $5 \%$ albedo.

\begin{tabular}{rrrrr}
\hline Detector & $\begin{array}{r}\text { Mean } \\
\text { (orbit) }\end{array}$ & $\sigma$ & $\begin{array}{r}\text { Mean } \\
\text { (ground) }\end{array}$ & $\begin{array}{r}\text { SNR } \\
\text { (at 5 \% albedo) }\end{array}$ \\
\hline 1 & 46.48 & 1.39 & 47 & 27.18 \\
2 & 41.25 & 1.43 & 42 & 26.28 \\
3 & 40.53 & 1.44 & 41 & 26.20 \\
4 & 41.71 & 1.41 & 42 & 27.01 \\
5 & 45.65 & 1.50 & 46 & 25.24 \\
6 & 39.61 & 1.39 & 40 & 27.08 \\
7 & 39.52 & 1.44 & 40 & 26.00 \\
8 & 36.84 & 1.43 & 37 & 26.21 \\
\hline
\end{tabular}

is about 0.7 counts at detector number 2) with the values obtained during the ground tests. The standard deviation of $100 \times 100$ IFOVs is the key parameter that determines the SNR value and shows quite a small number, less than 1.5. As a consequence, the derived SNR values are greater than 25 for all eight detectors. These performance results of COMS/MI are well within the user requirement (greater than 10) and are also comparable with the legacy instruments (Hillger and Schmidt, 2011). 
Table 3. PRNU of VIS detectors at $5 \pm 1 \%$ albedo. PRNU should be less than one-third of noise-equivalent delta radiance derived from SNR at $5 \%$ albedo $\left(0.8 \mathrm{~W} \mathrm{~m}^{-2} \mathrm{sr}^{-1} \mu \mathrm{m}^{-1}\right)$.

\begin{tabular}{rrr}
\hline Detector & $\begin{array}{r}\text { PRNU at } 5 \pm 1 \% \\
\text { albedo (side 1) }\end{array}$ & $\begin{array}{r}\text { PRNU at } 5 \pm 1 \% \\
\text { albedo (side 2) }\end{array}$ \\
\hline 1 & reference & reference \\
2 & 0.14 & 0.06 \\
3 & 0.13 & 0.06 \\
4 & 0.10 & 0.09 \\
5 & 0.16 & 0.02 \\
6 & 0.02 & 0.07 \\
7 & 0.04 & 0.12 \\
8 & 0.02 & 0.17 \\
All detectors & 0.09 & 0.08 \\
\hline
\end{tabular}

The image quality, especially for the imaging instrument such as COMS/MI, is also dependent on the relative channel characteristics among the eight detectors. For example, even though all eight detectors meet quality requirements such as $\mathrm{SNR}$, if relative performances are too different among the eight detectors, the overall image quality will be degraded. To detect the relative performance and mitigate the severe difference if there is any, the detector uniformity is estimated using the pixel-to-pixel response nonuniformity (PRNU) values. The PRNU values for COMS/MI visible channels are obtained from the difference between the detectors at $5 \%$ albedo using Eq. (2):

$\mathrm{PRNU}=\frac{1}{N_{5} \%} \sum_{N_{5} \%}\left(R_{\text {Det_R }}-R_{\text {Det } \_\mathrm{i}}\right) \leq \frac{R_{5} \%}{3 \times \mathrm{SNR}_{5} \%}$,

where $N_{5} \%$ denotes number of space look counts used for the calculation and $R_{\text {Det_R }}$ and $R_{\text {Det_i }}$ are the radiance values at the reference detector and the individual detector, respectively. Here, we use detector number 1 as our reference detector. As shown in Eq. (2), the user requirement for the PRNU value is less than one-third of the noise-equivalent delta radiance (NEdR), derived from the SNR value at $5 \%$ albedo.

The overall PRNU performance of side 1 and 2 of the visible-channel detectors (side 1 is the operational and side 2 is backup) are summarized in Table 3 . The performance values are obtained from the full-disk images taken during the $24 \mathrm{~h}$ of operation on 10 to 11 August and on $15 \mathrm{Au}$ gust for side 1 and side 2, respectively. The PRNU values of the seven other detectors are all well below the requirement $\left(0.8 \mathrm{~W} \mathrm{~m}^{-2} \mathrm{sr}^{-1} \mu \mathrm{m}^{-1}\right)$ by a significant margin. As shown in Table 2, detector number 5 has a slightly lower SNR value compared to detector number 1. Consequently, the PRNU value at detector number 5 is slightly larger than that of other detectors. However, the absolute number is considerably below the requirement, and thus it is concluded that the overall discrepancy introduced by the disparate SNR value is negligible. The quantitative results are also confirmed by the qualitative inspection of the visible channel images obtained

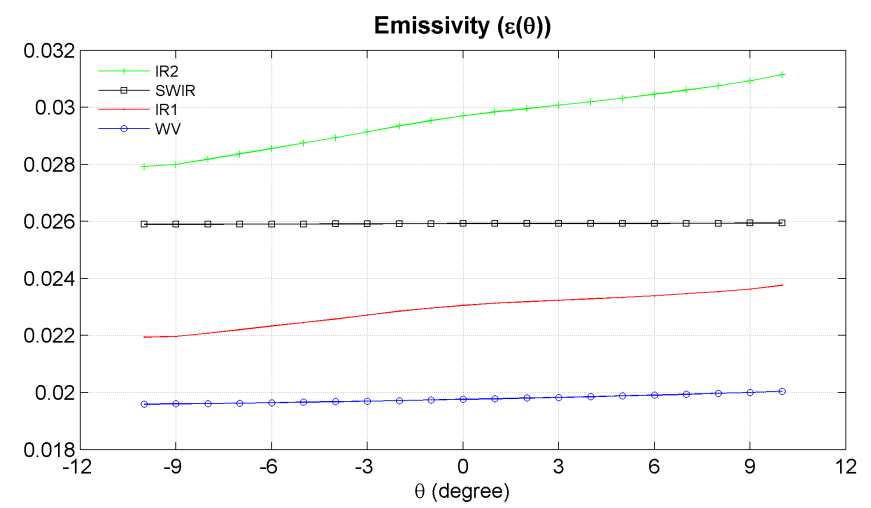

Figure 6. Scan mirror emissivity as a function of scan angle for all four channels. Zero degrees represents the nadir-looking position, negative signs represent the west direction, and positive signs the east direction from the center of the Earth disk. In operation, the scan angle dependence is fitted with a quadratic equation and applied in real time.

during the test period. However, the PRNU values during around local midnight are larger than the requirement, although this does not have a significant meaning. First of all, the visible-channel data obtained during this time of day are not used. Second, the PRNU values is derived before any correction of the stray light or direct sunlight intrusion effects, which significantly degrade the radiometric performances. The overall results concluded that there is no need of any additional processing such as the normalization of detector signals on the visible channels.

\subsection{Infrared channels}

The radiometric performance of infrared channels depends on many parameters such as the scan mirror emissivity, detector patch temperature in terms of absolute value and stability, accurate knowledge of the internal calibration target, control of the stray light, overall instrument alignment, etc. (Weinreb et al., 1997; Wu and Yu, 2013). The overall performance parameters such as NEdT and PRNU are the result of combined effects of all these parameters. Thus, here we introduce several activities conducted for COMS/MI to improve the overall performance and briefly summarize the IOT results.

For the infrared channels, one of the most well-known external source of uncertainty is the scan angle dependence on scan mirror emissivity (Weinreb et al., 1997; MacDonald, 1999). To mitigate this effect, a new coating material for the scan mirror was applied for COMS/MI, and the performance is evaluated using the space look count (Astrium EADS, 2011a), which is obtained by scanning the full width of the $\mathrm{E}-\mathrm{W}$ direction for the area well above the Earth disk edge. As shown in Fig. 6, the scan angle dependence is prominent in the IR1 and IR2 channels and it should be accounted for by the calibration equation. By using the space look count, 
Table 4. The noise performance of COMS/MI for each detector with the user-required specifications for two different conditions: 220 and $300 \mathrm{~K}$. The measurement values are given for each detector, although the requirement is the same for the same for each detector in the same channel.

\begin{tabular}{|c|c|c|c|c|c|}
\hline \multirow{2}{*}{ Band } & \multirow{2}{*}{ Detector } & \multicolumn{2}{|c|}{$220 \mathrm{~K}$} & \multicolumn{2}{|c|}{$300 \mathrm{~K}$} \\
\hline & & Measurement & Requirement & Measurement & Requirement \\
\hline \multirow{2}{*}{ SWIR } & A & 3.08 & \multirow{2}{*}{5.70} & 0.09 & \multirow{2}{*}{0.10} \\
\hline & B & 3.04 & & 0.08 & \\
\hline \multirow{2}{*}{ WV } & A & 0.27 & \multirow{2}{*}{0.86} & 0.04 & \multirow{2}{*}{0.12} \\
\hline & B & 0.28 & & 0.04 & \\
\hline \multirow{2}{*}{ IR1 } & A & 0.01 & \multirow{2}{*}{0.40} & 0.02 & \multirow{2}{*}{0.12} \\
\hline & B & 0.03 & & 0.02 & \\
\hline \multirow{2}{*}{ IR2 } & A & 0.16 & \multirow{2}{*}{0.48} & 0.05 & \multirow{2}{*}{0.20} \\
\hline & B & 0.15 & & 0.05 & \\
\hline
\end{tabular}

quadratic correction coefficients for the scan angle dependence are derived and incorporated into the calibration process (Astrium EADS, 2011c). To check the stability of the correction coefficients, the dependence is checked for a short time period, over $24 \mathrm{~h}$, and for a longer time period, separated by about 2 months, the first one on 16 August and the second one on 11 October. From the comparison, it is found that there is no significant temporal variability in the scan angle dependence of the scan mirror emissivity and thus in the scan angle correction parameters (Astirum EADS, 2011a). Thus, it is concluded that the emissivity correction as a function of scan angle is necessary for all infrared channels except for the SWIR channel, and the fixed correction coefficients are sufficient for a short and longer term, although it is recommended that regular monitoring be done for a longer-term drift.

Another well-known calibration uncertainty arises during the satellite midnight, when direct solar radiation can impinge on the internal blackbody. When this happens, the direct radiation interferes with the regulation of the blackbody temperature at a constant temperature and introduces error into the measured blackbody radiation through the reflection of the incident light by the imperfect blackbody. Thus, the overall calibration uncertainty increases and a corrective measure should be applied (Johnson and Weinreb, 1996). To correct for this effect, a sensitivity test for the dependence of the calibration slope on the different sets of temperatures of optics components - such as the scan mirror, primary and secondary mirror, and louvre - has been conducted. From the sensitivity tests, the closest correction parameter is obtained from the temperature of scan mirror for the SWIR channel and of the primary mirror for the other infrared channels. Thus, combining the daytime calibration slope and nighttime slope with the temperature information for the previous 5 days, the midnight calibration correction is successfully applied (Astrium EADS, 2011a).
After application of developed measures for the all identified uncertainty sources, the noise performances are derived. Table 4 shows the resultant NEdT values of the four infrared channels of COMS/MI at 220 and $300 \mathrm{~K}$, and corresponding user requirement specifications. For the estimation of the NEdT values, the following procedure is applied:

$\mathrm{NEdT}=(A+B \times T)-T_{\mathrm{ref}} ;$

$T$ is obtained by conversion of the Planck function,

$T=\frac{10^{6} h c}{\ln \left(\frac{2 \times 10^{24} h c^{2}}{\lambda_{\mathrm{c}}^{5} R}\right) \lambda_{\mathrm{c}} k}$,

where $h$ is the Planck constant $\left(6.62617 \times 10^{-34} \mathrm{~J} \mathrm{~s}\right), c$ is the speed of light in vacuum $\left(2.99792458 \times 10^{8} \mathrm{~m} \mathrm{~s}^{-1}\right), k$ is the Boltzmann constant $\left(1.38066 \times 10^{-23} \mathrm{~J} \mathrm{~K}^{-1}\right)$, and the $\lambda_{\mathrm{c}}$ is the central wavelength of the infrared channels. The band center wavelength can be derived either by finding a wavelength matching half of the area that the SRF covers with the wavelength or by estimating the SRF weighted-averaged wavelength ( $\mathrm{Wu}$ and $\mathrm{Yu}, 2013)$. Here we use the first approach to define the center wavelength. The coefficients $A$ and $B$ are the conversion coefficient between effective and brightness temperature. The reference temperature, $T_{\text {ref }}$, is $220 \mathrm{~K}$ for the space look count and $300 \mathrm{~K}$ for the blackbody radiance. The performance values given in Table 4 clearly show that the noise values of each detector and channel are less than the specification and meet the user requirement by a significant margin, especially at $220 \mathrm{~K}$. All eight detectors show that the NEdT values at $220 \mathrm{~K}$ are more than 2 times better than the requirement. There is a significant margin at the IR1 and IR2 channels even at $300 \mathrm{~K}$, although the SWIR and WV channels have a lesser margin.

The temporal variation of the radiometric performance is checked using the monthly mean variation of NEdT obtained during the IOT period and is summarized in Fig. 7. For the Earth scene temperature (Fig. 7a), other than the slightly larger value and variation shown in October at the SWIR 


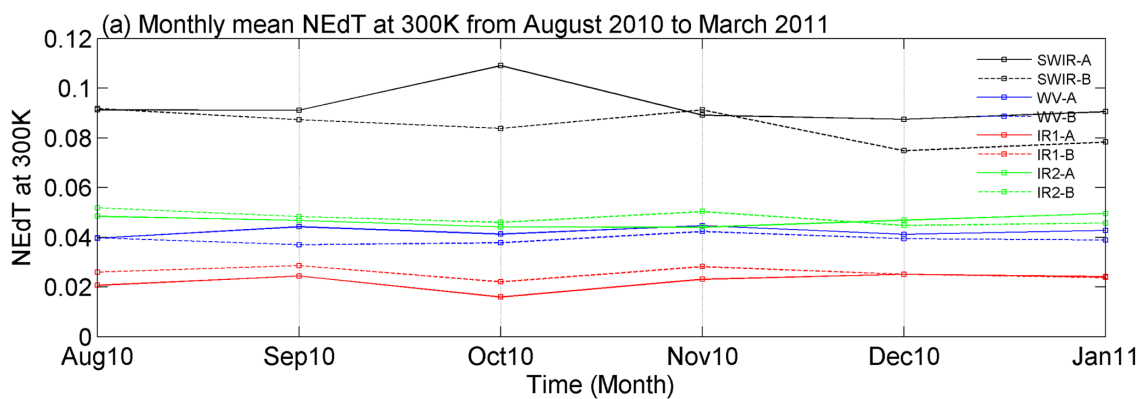

(b) Monthly mean NEdT at 220K from August 2010 to March 2011

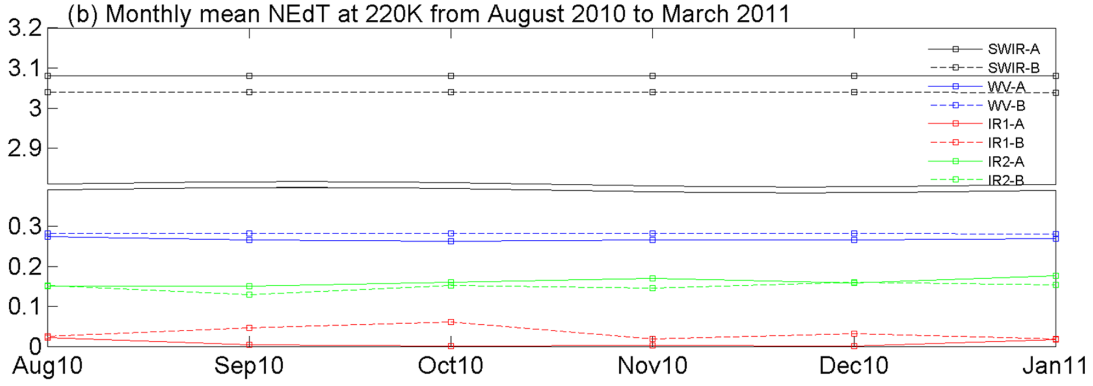

Figure 7. Monthly mean NEdT at $300 \mathrm{~K}$ (a) and $220 \mathrm{~K}$ (b) for four IR channels from August 2010 to January 2011.

channel, no significant temporal variation in the NEdT performance is detected. Other than at the SWIR channel, the channel-to-channel disparity seems slightly larger during the summertime, although the differences are well below the user requirement. However, at the SWIR channel, during October and December 2010 and January 2012, the difference is about $0.01 \mathrm{~K}$, which is still under the user requirement, although it is worth monitoring for any further degradations or fluctuations. The overall conclusion made with the Earth scene temperature is also applicable to the characteristics obtained for the space look temperature. However, it should be noted that the detector-to-detector difference at the SWIR channel increases to the level of about $0.05 \mathrm{~K}$, which is simply due to the sensitivity of temperature to the radiance at the lower brightness temperature.

Finally, Fig. 8 shows the diurnal variation of NEdT at $300 \mathrm{~K}$ for each IR detector derived from data obtained January 2011, which provides a sufficient number of data points. The overall conclusion from the monitoring is that there is no significant diurnal variation in the NEdT values nor the channel-to-channel difference. Again, the NEdT values in Fig. 8 are all within the range of the user requirement except for one of SWIR detectors, although its overall performance is within the specification (see Table 4). The mean difference between two detectors in the same channel is within 0.005 , except for SWIR with a value of around 0.01, for which the maximum difference during the day is at around 03:00 to 06:00 UTC (not shown). However, it should be noted that both the monthly mean (in October) and 3-hourly mean NEdT (12:00 UTC) of the SWIR channel at $300 \mathrm{~K}$ (as shown in Figs. 7 and 8) is slightly larger than the requirement $(0.1 \mathrm{~K})$, whereas the overall mean value is within the requirement. Thus, continuous monitoring and characterization of the SWIR channel is recommended.

When the NEdT values given in Table 4 are compared with those of the legacy instruments as shown in Table 5, the noise performance of COMS/MI is comparable to or slightly worse than the latest GOES series (i.e., GOES 13, 14, and 15), which utilize a single-solar-panel spacecraft configuration similar to the COMS configuration. However, the noise performances of COMS/MI are better than those of legacy instruments onboard the previous GOES series, which use a single solar panel and a balance boom on the opposite side of the spacecraft to where the imager is located (SS/Loral, 1996). The reflected solar radiation by the balance boom adds a heat source to the imager, and the regulated patch temperature was $94 \mathrm{~K}$ (during the winter solstice, $101 \mathrm{~K}$ for the other 6 months). However, in the case of COMS/MI, the operational patch temperature is about $85 \mathrm{~K}$ due to the design of the spacecraft, i.e., the single solar panel located on the other side of the MI instrument.

Although there are only two different detectors for each infrared channel (compared to the eight detectors for the visible channel), the PRNU values for each infrared channels are estimated. The obtained values at 220 and $300 \mathrm{~K}$ are all within the specification (not shown). The ratios of the measured PRNU-to-PRNU requirement (i.e., 0.0007 for SWIR; 0.007 for WV; and 0.005 and 0.008 for IR1 and IR2, respectively) are within about $30 \%$, except for SWIR, whose values reach up to $80 \%$ of the requirement at $300 \mathrm{~K}$. From these performance results, we conclude that the IOT test results for the operational infrared channels are within the user requirement; COMS/MI was commissioned on 1 April 2011, just 9 months after the launch of the satellite. 

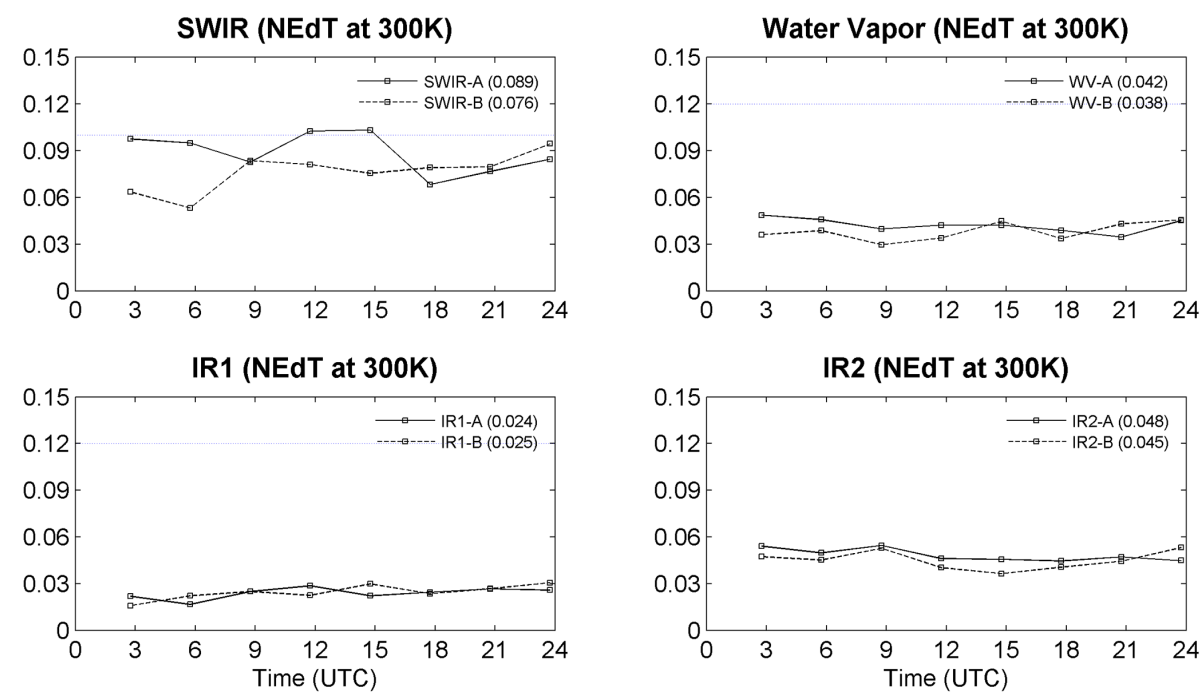

Figure 8. Diurnal variation of NEdT at $300 \mathrm{~K}$ of four IR channels for each detector in the channel during the January 2011. Diurnal mean of NEdT for each detector is in parentheses. Dashed lines indicate the requirement at a given channel in Table 4. Note that the requirement of NEdT of IR2 is 0.2. Data of full-disk images are presented in $3 \mathrm{~h}$ intervals.

Table 5. Comparison of COMS/MI noise performance with the legacy instruments onboard previous weather satellites. The IR2 channel is not available after GOES 12, which has the new carbon dioxide channel instead of the IR2 channel. The performance values of the legacy instrument are from Hillger and Schmit (2011). In the case of COMS/MI, the performance values are for detector number 1 and number 2.

\begin{tabular}{llllllllll}
\hline Band & COMS & G-15 & G-14 & G-13 & G-12 & G-11 & G-10 & G09 & G-08 \\
\hline SWIR & 0.070 & 0.063 & 0.053 & 0.051 & 0.13 & 0.14 & 0.17 & 0.08 & 0.16 \\
& 0.070 & & & & & & & & \\
WV & 0.080 & 0.170 & 0.18 & 0.14 & 0.15 & 0.22 & 0.09 & 0.15 & 0.27 \\
& 0.060 & & & & & & & & \\
IR1 & 0.060 & 0.059 & 0.06 & 0.053 & 0.11 & 0.08 & 0.20 & 0.07 & 0.12 \\
& 0.040 & & & & & & & & \\
IR2 & 0.110 & & & & & 0.20 & 0.24 & 0.14 & 0.20 \\
& 0.120 & & & & & & & & \\
\hline
\end{tabular}

\section{Summary}

The first geostationary Earth observation satellite of Korea, COMS, was launched on 27 June 2010. Soon after the satellite's arrival at the operational orbit of $128.2^{\circ} \mathrm{E}$, an inorbit test (IOT) was started that lasted about 8 months. The tests for the operational use of the meteorological imager, COMS/MI, started with the acquisition of the first visiblechannel images on 12 July 2010 . With the successful test for acquisition, processing, and dissemination of the data, the overall signal-processing chains were verified. During the outgassing period, functional tests of COMS/MI such as the command execution, mode switching, redundancy selection of the MI channels, coordinate verification, and scan time were conducted without any significant problems. During the tests, several interesting images such as the series of the Moon and albedo monitor were also acquired.
After successful acquisition of the four infrared channels, a full functional and performance test was started. This also included the fine tuning of the iimage-navigation-andregistration (INR) algorithms and extensive verification of the INR performances at system level. All of the functional and performance estimations obtained during the IOT were all within the specifications and met the requirements for operational applications. The noise values were all comparable with the most recent series of legacy instruments and better than the previous legacy instruments. More importantly, the designed performances were achieved within 8 months after the launch of the satellite (the original plan was for 6 months). With the successful performance of the IOT, it was concluded that COMS and its payloads were ready to full service, and its service started from 1 April 2011.

The data acquired during the in-orbit test will play an important role in understanding the nature and characteristics of the current and future COMS/MI data. Thus it is highly 
recommended to process with all the updated parameters and make the results available to users. It would be quite important to make these valuable data available to as many users as possible for the further utilization of COMS/MI data. New and innovative perspectives on the first set of observed data would generate a new and better way for the characterization of a new instrument planned for the next generation of COMS. Moreover, in spite of the satisfactory test results from IOT, it is highly possible that COMS/MI will experience unexpected changes in performance or degradation. Thus, it is highly recommended to monitor the longterm changes, the diurnal variation, and the status of the performance of COMS/MI. Inter-satellite comparison with the well-calibrated reference instrument (Goldberg et al., 2011; Hewison et al., 2013) is a good activity for that purpose. 


\section{Appendix A: COMS/MI and IOT results}

\section{A1 COMS/MI}

The first multi-purpose geostationary observation satellite of Korea, known as COMS (Communication, Ocean, and Meteorological Satellite), was launched on 27 June 2010 in KST, at Guiana Space Center at Kourou in French Guiana using the Ariane-5 launch vehicle. At $25 \mathrm{~min}$ after the launch, the satellite successfully attained the transition altitude of $250 \mathrm{~km}$. From that point on, COMS was lifted into the geostationary orbit by using the LAE (liquid apogee engine) of the satellite, and it successfully arrived at its service orbit of $128.2^{\circ} \mathrm{E}$ on 6 July 2010, as planned.

As can be seen from its name, COMS is designed to perform three major missions: operational weather observation by using a meteorological imager (MI), oceanography observation with the Geostationary Ocean Color Imager (GOCI), and space proofing of a Ka-band telecommunication payload. These three missions are supported by three different Korean governmental entities such as the Korea Meteorological Administration (KMA), the Ministry of Oceans and Fisheries (MOF), and finally the Korea Communication Commission. The project was developed under the overall responsibility of the Korea Aerospace Research Institute (KARI), supported by the Ministry of Science, ICT and Future Planning (MISP). The designed mission lifetime of COMS is 7 years, which is longer than that of usual geostationary meteorological satellite missions, about 5 years.

Soon after the arrival of the satellite at its service orbit, the in-orbit test (IOT) was started, which lasted for about 8 months. During the IOT, not only the space component of the program but also the ground facilities were tested, adjusted, demonstrated, and validated. The test was conducted by Astrium EADS, which has the overall responsibility of satellite manufacturing with the help from KARI, KMA, KIOST (Korea Institute of Ocean Science and Technology, responsible for the development and operation of the ocean mission), and ETRI (Electronic and Telecommunication Research Institute, which developed the telecommunication payload and will operate the payload). During the test, all of the command and communication activities were conducted at the COMS CDA (Command and Data Acquisition) facility at KARI with the help of the participating organizations. The meteorological data processing facility at the National Meteorological Satellite Center (NMSC) of KMA was developed as the backup station for the COMS CDA and primary station for real-time processing of the MI data. During the IOT, the NMSC facility was also used for the backup operation and real-time MI data processing.

To meet the user requirement, COMS/MI was designed with three major parts: an electronics, optics, and power supply module. The major functions of the electronics module are command, control, and data processing. The power supply module provides necessary electric power for the

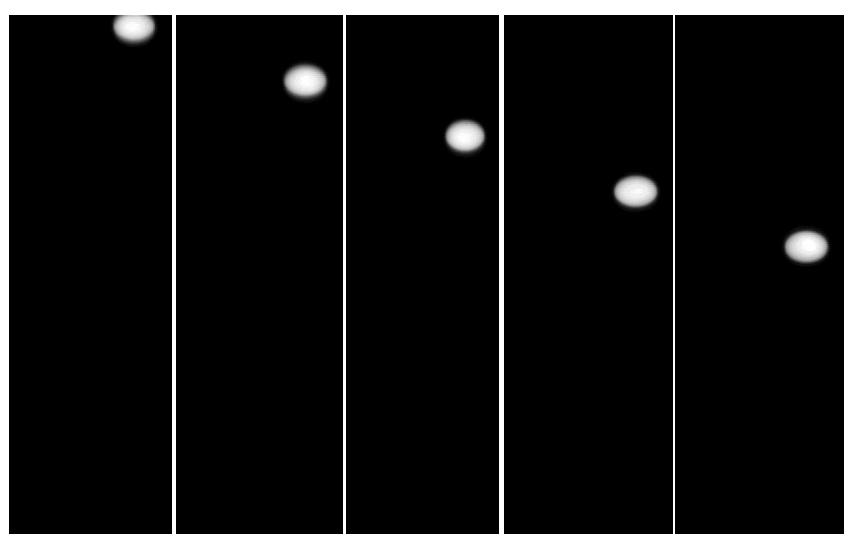

Figure A1. Sequential images of the Sun captured by one of visible channels through the albedo monitor onboard COMS/MI.

electronics and sensor module by interfacing with the spacecraft. The sensor module is the main component for the actual observation and has major components such as a scan assembly, telescope, spectrometer, detectors with a passive cooler, and an internal calibration target (a blackbody) and albedo monitor. COMS/MI uses a Cassegrain-type telescope with primary and secondary reflecting mirrors that have a diameter of $31.1 \mathrm{~cm}$ and $3.8 \mathrm{~cm}$, respectively. The two-axis gimbaled scanner sweeps in the $\mathrm{E}-\mathrm{W}$ direction with a width of $8 \mathrm{~km}\left(\mathrm{~N}-\mathrm{S}\right.$ direction) in 20.0 degree $^{-1}$ optical speed. This results in a scanning time of about $27 \mathrm{~min}$ to cover the full disk of Earth, which covers a field of regard of about $17.7^{\circ}$ for $\mathrm{N}-\mathrm{S}$ and $\mathrm{E}-\mathrm{W}$ directions.

The incident radiation coming through the telescope is separated by a series of beam splitters before entering into the detectors. The first dichroic beam splitter reflects infrared radiation into the next infrared dichroic beam splitter, while it transmits visible radiation into the eight visible detectors (corresponding $1 \mathrm{~km}$ spatial resolution), which are operated at ambient temperature. The infrared radiation undergoes further separation into the specified band wavelengths through the series of the beam splitter and band-pass filters. There are two detectors for each channel (corresponding to $4 \mathrm{~km}$ spatial resolution), which are mounted on the same detector patch. The operational temperature required for the IR detectors is cryogenic temperature and is obtained by a passive radiant cooler which has three parts: the vacuum housing, the radiator, and the patch. To achieve the maximum performance of the passive cooler, the spacecraft is designed to minimize interference to the cooler by securing a full field of regard of the vacuum housing and to prevent external light from entering the cooler.

For the radiometric calibration, COMS/MI has two components: a blackbody for the infrared channels and an albedo monitor for the visible channel. The blackbody is installed on the baseplate of the sensor module, while the albedo monitor is located underneath the sensor module. As the blackbody 
is used for the warm target, its temperature needs to be regulated at a fixed temperature with a certain range of variability. This is done by means of heat pipes which regulate the blackbody temperature within the specification. For a better temperature reading of the blackbody, there are eight thermistors embedded under the blackbody surface. Another target for the infrared channel calibration, the cold target, is provided by space, which has a fairly stable background temperature of about $2.7 \mathrm{~K}$. Thus, for the infrared calibration, the scan mirror needs to look at the blackbody and space.

However, there is no true onboard calibration target for the visible channel. Thus, for absolute calibration of the visible channel, other methods need to be resorted to, such as vicarious calibrations using the reference target method (Wu et al., 2011; Chun et al., 2012) or satellite-to-satellite intercomparison (Chander et al., 2013). However, there is one component, the albedo monitor, which can be used for the monitoring of an overall trend of telescope mirrors (excluding the scan mirror) and spectrometer characteristics. It reflects solar radiation into the optical chain (to the primary mirror) when the satellite is in correct alignment with the Sun (it usually occurs early in the satellite morning hours). As only a small portion of the reflected solar radiation from the albedo monitor shines on the primary and secondary mirror, the signal is only detected by one specific detector among the eight detectors (refer to Fig. A1). Due to the partial exposure of the optical chain to solar radiation, the measured data are only used for the monitoring of an overall trend, not for an absolute calibration of the visible channels.

One of the most important overall sensor characteristics is the SRF, which represents the weights applied to the the conversion of input signal to detector output. SRFs for the infrared channels for the two different sides of detector sets are obtained before the launch of the satellite. Although the band center and width are quite similar, there are differences (see Fig. 1). For example, the bandwidth of the water vapor channel for COMS/MI is much narrower than for the GOES series. In the case of the SWIR channel, the center wavelength of COMS/MI is slightly shorter than the GOES series, with the expectation of less contamination due to $\mathrm{CO}_{2}$ absorption. The COMS/MI SRFs are available at the KMA/NMSC webpage (http://nmsc.kma.go.kr/html/ homepage/ko/information/News/searchNews.do).

\section{A2 IOT tests and functional performance}

During the IOT, COMS/MI underwent a series of tests and performance evaluations. To meet the purposes of COMS/MI IOT test, activities includes the verification of the command and control of the MI, such as command execution, mode switching, redundancy selection of the MI channels, and monitoring of the instrument through direct telemetry. The test also includes verification of radiometric performances, such as visible and infrared channel radiometry, visiblechannel response verification using the albedo monitor, and

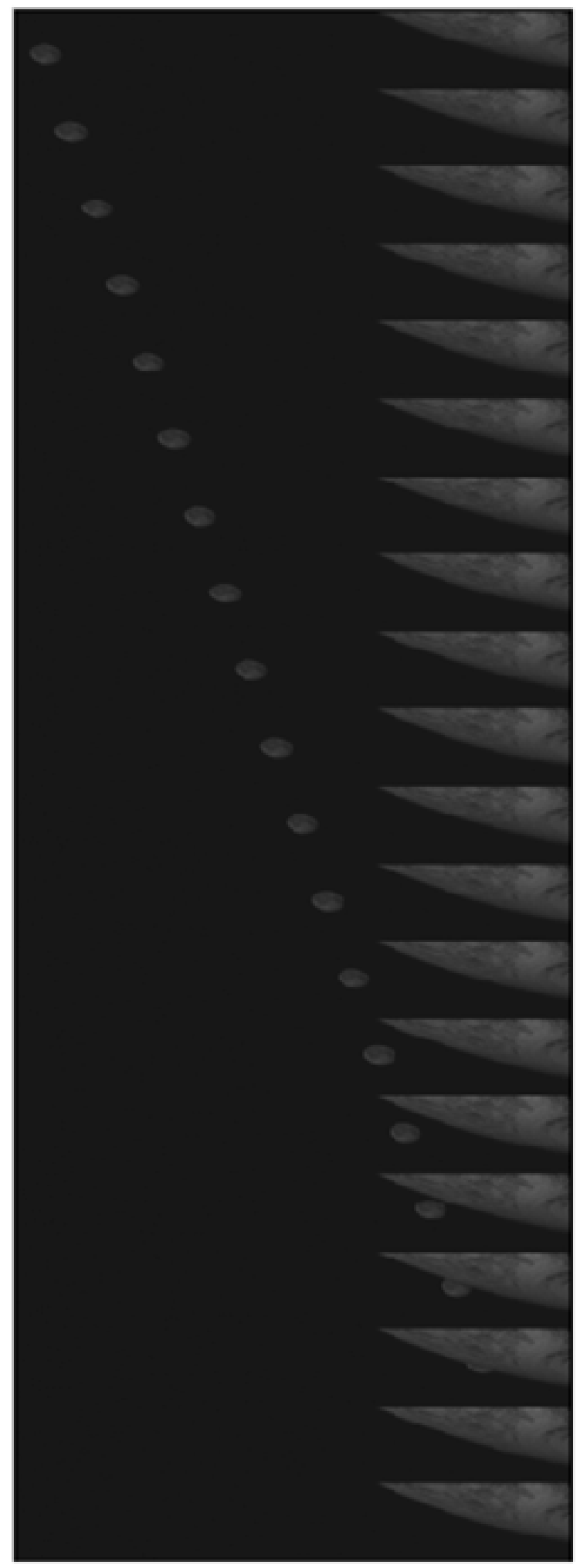

Figure A2. Series of images taken for lunar observation on 28 July to test the scan mechanism and accuracy of the coordinate system. 
(a)

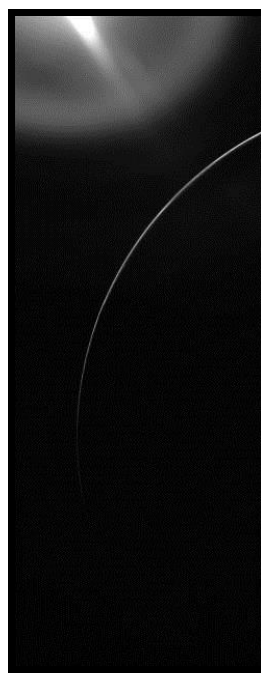

(b)

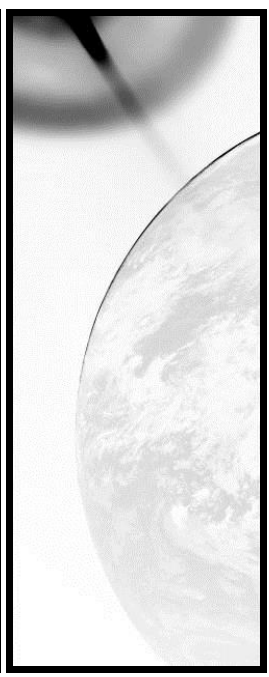

(c)

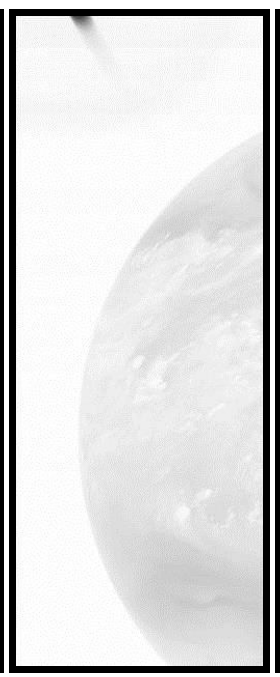

(d)

(e)

Figure A3. Effect of solar intrusion on the optical cavity of COMS/MI for different channels: (a) visible, (b) shortwave infrared, (c) water vapor, and the two infrared window channels (d) and (e) on 20 August 2010.

verification of the continued image acquisition throughout the Earth eclipse time period. It also includes the fine tuning of the image-navigation-and-registration (INR) algorithms and its extensive verification of performances at system level. For COMS/MI, there are several important IOT stages to be mentioned here which depended on the operational phases of IOT.

First of all, during the launch and orbit-raising operation, the MI is in standby mode, but MI housekeeping telemetry data are available via the satellite for the health check and monitoring of COMS/MI. Because COMS/MI does not have any protective cover to prevent direct solar intrusion into the primary mirror of the instrument, a careful plan to prevent direct viewing of the Sun was prepared and exercised. Other than that, IOT activity during this phase is limited to housekeeping telemetry monitoring.

After the arrival at the service orbit and subsequent successful acquisition of the first visible images, several functional tests were conducted for the visible channel and the instrument itself. For example, image acquisition time and scan rate are tested and verified. When COMS/MI is first commanded to take images of $19.48^{\circ} \mathrm{E}-\mathrm{W}$ and $17.75^{\circ} \mathrm{N}-$ $\mathrm{S}$ (viewing angles at the geostationary orbit), it takes slightly over (about $3 \mathrm{~s}$ ) $27 \mathrm{~min}$ (which is the user requirement). Thus the $\mathrm{E}-\mathrm{W}$ scan width is reduced to $19.27^{\circ}$, which results in the reduction of the acquisition time to $26 \mathrm{~min}$ and $48 \mathrm{~s}$, satisfying the user requirement. Another functional test was to test the scan mechanism and coordination accuracy. In order to do that, a series of Moon images is acquired with the anticipation of visible-channel calibration (Grant et al., 2001; Wu et al., 2006); these Moon images could be used as the baseline for further long-term monitoring of sensor degradation. Figure A2 shows the first series of lunar image taken in 28 July, 16 days after the start of visible-channel observation. The series of images clearly indicate the accuracy of the coordinate system and proper functioning of the scan mechanism and its stability. Finally, a test for the model of the orientation of the satellite, Sun, and Earth, as well as the scan mechanism, is conducted by passing the Sun image through the albedo monitor. Figure A1 shows the sequence images of the Sun taken at one of the visible channels during the test.

With the successful acquisition of all five channel images, further functional and performance tests were conducted. One of the most important additional functional tests was the accuracy of the INR algorithm. Its accuracy determines not only the image quality but also the quantitative products, such as the atmospheric motion vector, and daily or weekly sea surface temperature, which use the consecutive series of images. With several adjustments to the pre-launch algorithm and refinement of key algorithm parameters through the utilization of actual observation data, the INR performance achieved is quite a success, and the INR accuracy is smaller than one infrared image pixel (Astrium EADS, 2011b). The success is partly due to the successful stabilization of the spacecraft itself (a detailed description of the processes and results is under preparation) and the maximum utilization of available landmarks within the field of regard of COMS/MI (Astrium EADS, 2011b).

One of the important improvements with COMS/MI over the legacy spacecraft and instruments was the expectation of continuous operation during the eclipse period and resilience to direct solar intrusion into the optical cavity. With this improvement, COMS/MI could provide a continuous 
observation capability throughout the satellite midnight. As a demonstration, a test of this capability was conducted on 20 August 2010, and the results are shown in Fig. A3. As the Sun is on the opposite side of the surface of the Earth facing the satellite, the visible-channel image shows the dark Earth, with the Sun shining brightly at the top of the image. During this time of day, the visible channel does not provide any meaningful information and the solar intrusion does not have significant meaning for the operational use of data. However, as the four infrared images provide meaningful information, it is important to minimize or characterize the solar intrusion. As shown in Fig. A3, all four infrared images show meaningful information, although there is an affected area by solar intrusion. The effect is amplified in the SWIR image, simply due to the higher radiation sensitivity of the SWIR channel. Consequently, the effect is much weaker at the other channels such as at the IR1 and IR2 channels. From the results, we were able to verify that the interruption of operation due to the so-called keep-out zone could be minimized. 
Acknowledgements. The authors are indebted to all the hardworking people who took part in the in-orbit test; it is difficult to name each person from Astrium of EADS, KARI, and NMSC, but without their devotion and expertise, the successful results of IOT would not have been possible or would have been delayed much longer than it actually took. Critical reviews and constructive suggestions by two anonymous referees were a great help in improving the manuscript and are greatly acknowledged. Dohyeong Kim is supported by the National Meteorological Satellite Center (project no. 153-3100-3137-302-210-13). M. H. Ahn is partially supported by the Eco Innovation Program of KEITI (2012000160002).

Edited by: F. Prata

\section{References}

Astrium EADS: COMS Meteo-Imager In-Orbit Test Report, including Image Quality Verification Report, COMS.RP.00345.DP.T.ASTR, 169 pp., $2011 \mathrm{a}$.

Astrium EADS: COMS INR In-Orbit Test Report, including Image Quality Verification Report, COMS.RP.00347.DP.T.ASTR, 52 pp., $2011 b$.

Astrium EADS: Meteo-Imager Radiometric Model, COMS.TN.00116.DP.T.ASTR, 133 pp., 2011c.

CGMS: CGMS global contingency plan, WMO Space Programme, 22 pp., available at: https://www.wmo.int/pages/prog/ sat/documents/CGMS_Contingency-Plan-2007.pdf (last access: 5 August 2014), 2007.

Chander, G., Hewison, T. J., Fox, N., Wu, X., Xiong, X., and Blackwell, W. J.: Overview of intercalibration of satellite instruments, IEEE Trans. Geosci. Remote Sens., 51, 1056-1080, 2013.

Chun, H. W., Sohn, B. J., Kim, D. H., Ahn, M. H., and Ou, M. L.: Solar channel calibration using desert targets in Australia: Application to the MTSAT-1R, J. Meteo. Soc. Japan, 90, 191205, doi:10.2151/jmsj.2012-203, 2012.

Goldberg, M., Ohring, G., Butler, J., Cao, C., Datla, R., Doelling, D., Gaertner, V., Hewison, T., Iacovazzi, B., Kim, D., Kurino, T., Lafeuille, J., Minnis, P., Renaut, D., Schmetz, J., Tobin, D., Wang, L., Weng, F., Wu, X., Yu, F., Zhang, P., and Zhu, T.: The global space-based inter-calibration system (GSICS), Bull. Am. Meteorol. Soc., 92, 468-475, 2011.

Grant, I. F., Kieffer, H. H., Stone, T. C., and Anderson, J. M.: Lunar calibration of the GMS-5 visible band, Proceedings of the International Geophysics and Remote Sensing Symposium 2001, 4, 2769-2771, 2001.
Hewison, T. J., Wu, X. Yu, F., Tahara, Y., Hu, X., Kim, D., and König, M.: GSICS inter-calibration of infrared channels of geostationary imagers using Metop/IASI, IEEE Trans. Geosci. Remote Sens., 51, 1160-1170, 2013.

Hillger, D. W. and Schmit, J. J.: The GOES-15 Science Test: Imager and Sounder Radiance and Product Validations, NOAA TR NESDIS 104, 101 pp., Washington DC, 2011.

Johnson, R. X. and Weinreb, M.: GOES-8 Imager midnight effects and slope correction, Proc. SPIE, 2812, 596-607, 1996.

Kim, J., Yoon, J. M., Ahn, M. H., Sohn, B. J., and Lim, H. S.: Retrieving aerosol optical depth using visible and mid-IR channels from geostationary satellite, MTSAT-1R, Int. J. Remote Sens., 29, 6181-6192, 2007.

MacDonald, M. E.: Alternatives to $\mathrm{SiO}_{x}$ for protective scan mirror coatings in remote sensing instruments, Proc. SPIE 3750, Earth Observing Systems IV, 309, 309-318, doi:10.1117/12.363524, 1999.

Menzel, P. and Purdom, J.: Introducing GOES-I: The First of a New Generation of Geostationary Operational Environmental Satellites. Bull. Amer. Meteor. Soc., 75, 757-781, doi:10.1175/15200477(1994)075<0757:IGITFO>2.0.CO;2, 1994.

Purdom, J. and Menzel, P.: Evolution of satellite observation in the United States and their use in meteorology, Historical Essays on Meteorology 1919-1995, edited by: Fleming, J. R., American Meteorological Society, 99-156, 1996.

Schmetz, J., Pili, P., Tjemkes, S., Just, D., Kerkmann, J., Rota, S., and Ratier, R.: Supplement to An Introduction to Meteosat Second Generation (MSG), Bull. Am. Meteorol. Soc., 83, 991-991, doi:10.1175/BAMS-83-7-Schmetz-1, 2002.

SS/Loral: GOES I-M Databook, DRL 101-08, Rev. 1, 186 pp. 1996.

Weinreb, M. P., Jamison, M., Fulton, N., Chen, Y., Johnson, J. X., Bremer, J., Smith, C., and Baucom, J.: Operational calibration of Geostationary Operational Environmental Satellite-8 and -9 imagers and sounders, Appl. Optics, 36, 6895-6904, 1997.

Wu, X. and Yu, F.: Correction for GOES Imager Spectral Response Function Using GSICS. Part I: Theory. IEEE Trans. Geosci. Remote Sens., 51, 1215-1223, 2013.

Wu, X., Stone, T. C., Yu, F., and Han, D.: Vicarious calibration of GOES Imager visible channel using the Moon, Proc. SPIE, 6296 , 62960Z, doi:10.1117/12.681591, 2006.

Wu, X., Qian, H., Yu, F., and Beck, T.: Vicarious calibration of GOES visible channel using GOME-2, IGARSS 2011, 10331035, 2011. 